\title{
MicroRNA-100 functions as a tumor suppressor in cervical cancer via downregulating the SATB1 expression and regulating AKT/mTOR signaling pathway and epithelial-to-mesenchymal transition
}

\author{
CUIPING HUANG ${ }^{1}$, XIAOBO QIN $^{2}$, NA ZHAO $^{3}$, HUIJING JIN $^{4}$, SHUANGJUN ZHANG ${ }^{5}$ and HAIYAN YANG ${ }^{6}$ \\ ${ }^{1}$ Department of Gynaecology, The Second Hospital of Shandong University, Jinan, Shandong 250033; \\ ${ }^{2}$ Department of Obstetrics and Gynecology, Zhangqiu Maternity and Child Care Hospital, Jinan, Shandong 250200; \\ ${ }^{3}$ Department of Imaging, People's Hospital of Rizhao, Rizhao, Shandong 276800; ${ }^{4}$ Ward Department, \\ The People's Hospital of Zhangqiu Area, Jinan, Shandong 250200; ${ }^{5}$ Department of Radiology, \\ The People's Hospital of Zhangqiu Area, Jinan, Shandong 250200; ${ }^{6}$ Department of Interventional Medicine, \\ The Second Hospital of Shandong University, Jinan, Shandong 250033, P.R. China
}

Received May 17, 2019; Accepted March 3, 2020

DOI: $10.3892 / \mathrm{ol} .2020 .11686$

\begin{abstract}
Cervical cancer (CC) is a common malignant tumor among women worldwide, remaining the fourth most frequent cause of cancer death in women. Currently, microRNA (miRNA) is a prevalent topic in tumor-related research. The present study focused on the mechanisms of miR-100 in CC progression. qRT-PCR analysis revealed that the miR-100 expression was notably decreased in $\mathrm{CC}$ tissues. In addition, miR-100 downregulation was confirmed to be significantly related to the malignant clinicopathologic features of $\mathrm{CC}$ patients. Furthermore, miR-100 overexpression was also verified to significantly repress $\mathrm{CC}$ cell proliferation, migration and invasion abilities through modulating the AKT/mTOR signaling pathway and epithelial-to-mesenchymal transition. Bioinformatics analysis and luciferase reporter assay identified that special AT-rich sequence-binding protein 1 was a functional target for miR-100 in CC cells. Moreover, miR-100 overexpression was found to markedly repress the $\mathrm{CC}$ tumor growth in vivo. In conclusion, the above results revealed that miR-100 functioned as a cancer suppressor in CC progression and may provide insights into the novel therapeutic target for CC treatment.
\end{abstract}

\section{Introduction}

Cervical cancer (CC) is a prevalent gynecological malignancy with poor prognosis globally (1). In recent years, despite

Correspondence to: Dr Haiyan Yang, Department of Interventional Medicine, The Second Hospital of Shandong University, 247 Beiyuan Street, Jinan, Shandong 250033, P.R. China E-mail: hy0971046ty@163.com

Key words: cervical cancer, miRNA-100, SATB1, AKT/mTOR, epithelial-to-mesenchymal transition improvements in the diagnosis and therapeutic strategies for $\mathrm{CC}$, patients suffering from CC still presented metastasis and relapse, which remain the main reasons for tumor-related deaths (2). However, the underlying mechanisms of CC development have not yet been sufficiently elucidated. Therefore, further understanding of the mechanism on CC occurrence and development is urgently imperative to identify novel diagnostic and therapeutic biomarkers for CC patients. Emerging literature has demonstrated that microRNAs (miRNAs) are emerging as tumor regulators of multiple tumors $(3,4)$.

miRNAs may serve as key regulators of gene expression via targeting 3'-untranslated regions (3'-UTRs) of target genes, leading to the inhibition of protein translation or mRNA degradation. Moreover, recently, miRNAs have been verified to play pivotal functions in various events which are involved in oncogenesis, such as cell apoptosis, proliferation, survival, and metastasis, through repressing expression of their targets. For example, Fang et al (5) proposed that miR-21 enhanced triple-negative breast cancer cell proliferation and invasion through regulating PTEN; Xiao et al (6) reported that miR-144 suppressed colorectal cancer proliferation and migration through GSPT1; Yang et al (7) claimed that miR-203 inhibited gastric carcinoma cell proliferation, migration and invasion via targeting Slug. However, the precise functions of miR-100 in $\mathrm{CC}$ require further investigation. Therefore, the present study was performed to confirm the roles of miR-100 in CC carcinogenesis.

Epithelial-to-mesenchymal transition (EMT) is a notable process involved in tumor-associated metastases and invasion (8). In the progress of EMT, obvious changes on cell adhesion, polarities, and motile property have been confirmed. In general, EMT is typically featured by the upregulation of mesenchymal marker and downregulation of epithelial marker (9). Moreover, EMT is considered to be one of the critical steps in the metastatic cascades of multiple malignant tumors, including hepatocellular carcinoma (10), prostate 
carcinoma (11) and renal cell carcinoma (12). In addition, the AKT/mTOR signalling pathway has essential roles in basic cellular processes, including cell apoptosis, differentiation and growth, exerting oncogenic functions in tumorigenesis of different malignancies $(13,14)$. Thus, in the present study, it was investigated whether miR-100 regulated $\mathrm{CC}$ progression through regulation of EMT and AKT/mTOR pathway.

Special AT-rich sequence-binding protein 1 (SATB1) is a nuclear matrix-associated protein and implicated in regulating tissue-specific gene expression, having emerged as a novel modulator of oncogenic pathways (15). SATB1 has been reported to be involved in the metastasis and growth of numerous malignant tumors. For example, Qi et al (16) indicated that SATB1 promoted EMT and metastases in prostate cancer; Li et al (17) found that SATB1 facilitated tumor oral squamous cell carcinoma metastases and invasiveness; studies by Pan et al (18) demonstrated that SATB1 was correlated with metastasis and progression of breast carcinoma. These studies suggested that SATB1 exerted oncogenic roles in tumor progression. However, the detailed roles of SATB1 in CC still need to be further elucidated.

\section{Patients and methods}

CC tissue specimens. Fifty-eight pairs of $\mathrm{CC}$ tissues and matched adjacent normal tissues were collected from CC patients who underwent surgical resection in the Second Hospital of Shandong University (Jinan, China) between April 2015 and October 2017. The CC patients received no treatments before tissue collection. All patients involved in the present study provided written informed consent. The fresh tissue sample was frozen in liquid nitrogen immediately, then stored at $-80^{\circ} \mathrm{C}$ for further assays. The present study was approved by the Ethics Committee of the Second Hospital of Shandong University.

CC cell lines and cell cultures. Human CC cells (C-33A, HeLa, SiHa and Ca-Ski) and normal cervical epithelial cell line (Ect1-E6E7) were purchased from the Committee on Type Culture Collection of the Chinese Academy of Sciences (Shanghai, China). All the cells were maintained in Dulbecco's modified Eagle's medium (DMEM) with $10 \%$ fetal bovine serum (FBS) in a humidified incubator containing $5 \% \mathrm{CO}_{2}$ at $37^{\circ} \mathrm{C}$.

Cell transfections. miR-100 mimics, inhibitor or negative controls (NC) were obtained from GenePharma. Lipofectamine 2000 (Invitrogen; Thermo Fisher Scientific, Inc.) was utilized to transfect them into $\mathrm{CC}$ cells according to the manufacturer's proposal.

$q R T-P C R$. Total RNAs were isolated from the cultured cells and tissue specimens using TRIzol reagent (Invitrogen; Thermo Fisher Scientific, Inc.), followed by reverse transcription into cDNA by PrimeScript RT reagent kit (Takara Biotechnology, Co., Ltd.). qRT-PCR was conducted with SYBR $^{\circledR}$-Green PCR Master Mix (Takara Biotechnology, Co., Ltd.) on an ABI 7500 system (Applied Biosystems). The relative expression levels of genes were detected with the $2^{-\Delta \Delta \mathrm{Ct}}$ method. Expression of candidate genes was normalized to
Table I. Primer sequences for qRT-PCR.

\begin{tabular}{lc}
\hline Primer & \multicolumn{2}{c}{ Sequence } \\
\hline miR-100 & F: 5'-ACACTCCAGCTGGGAACCCGTAGAT \\
& CCGAAC-3' \\
miR-100 & R: 5'-TGGTGTCGTGGAGTCG-3' \\
U6 & F: 5'-CTCGCTTCGGCAGCACA-3' \\
U6 & R: 5'-AACGCTTCACGAATTTGCGT-3' \\
SATB1 & F: 5'-GTGGAAGCCTTGGGAATCC-3' \\
SATB1 & R: 5'-CTGACAGCTCTTCTTCTAGTT-3' \\
GAPDH & F: 5'-GGCCAAGGTCATCCATGACAA-3' \\
GAPDH & R: 5'-TCTTCTGACACCTACCGGGGA-3'
\end{tabular}

F, forward; R, reverse; U6, small nuclear RNA, snRNA; GAPDH, glyceraldehyde-3-phosphate dehydrogenase; SATB1, special AT-rich sequence-binding protein 1 .

that of glyceraldehyde-3-phosphate dehydrogenase (GAPDH) whereas U6 was an endogenous control for miR-100. The sequences of the primers are shown in Table I.

Immunohistochemistry (IHC). For IHC assays, CC tissues and adjacent normal tissues were formalin-fixed, paraffin-embedded and cut into $4 \mu \mathrm{m}$ sections. Then, the slides were deparaffinized in xylene and rehydrated through a descending series of ethanol. Antigen retrieval was performed with citrate buffer in microwave oven. Subsequently, slides were blocked with $3 \%$ hydrogen peroxidase in methanol and $10 \%$ goat serum. Slides were incubated with SATB1 antibody (1:200; ab109122; Abcam) overnight at $4^{\circ} \mathrm{C}$ and then with HRP-labeled goat anti-rabbit IgG (1:2,000, ab205718; Abcam) for $30 \mathrm{~min}$ at room temperature. The slides were stained with $\mathrm{DAB}$ as the chromogen and counterstained with hematoxylin. Then, a bright-field microscope (Olympus BX50; Olympus Corporation) was used to image and analyze the slides. Five visual fields were randomly chosen from each section by double-blind method. The double-blind method was utilized to randomly select 5 visual fields from each section. The expression levels were determined following the ratio of positive cells:stained cells. The cells $<25 \%$ were considered as negative $(-)$ while the ratio $>25 \%$ was positive $(+)(19,20)$.

Cell proliferation assay. MTT assays were carried out to assess the proliferation abilities of CC cells with different transfections. Briefly, transfected CC cells were plated into 96-well plate and incubated for $0,24,48$ or $72 \mathrm{~h}$ at $37^{\circ} \mathrm{C}$. Then, each well was added with MTT solution $(5 \mathrm{mg} / \mathrm{ml}, 10 \mu \mathrm{l})$ and incubated for $4 \mathrm{~h}$. Subsequently, dimethyl sulfoxide (DMSO) was added to each well to dissolve the formazan crystals. Finally, the $\mathrm{OD}_{490}$ was measured using the Multiskan Spectrum equipment (Thermo Fisher Scientifc, Inc.).

5-Bromo-2'-deoxyuridine (BrDU) assay. Cell proliferation rate was also assess by measuring the BrdU immunofluorescence incorporation of DNA during mitosis using the BrdU assay (Sigma-Aldrich; Merck KGaA) in accordance with the manufacturer's instructions. Cells $\left(1 \times 10^{4}\right)$ were cultured in 24 -well 

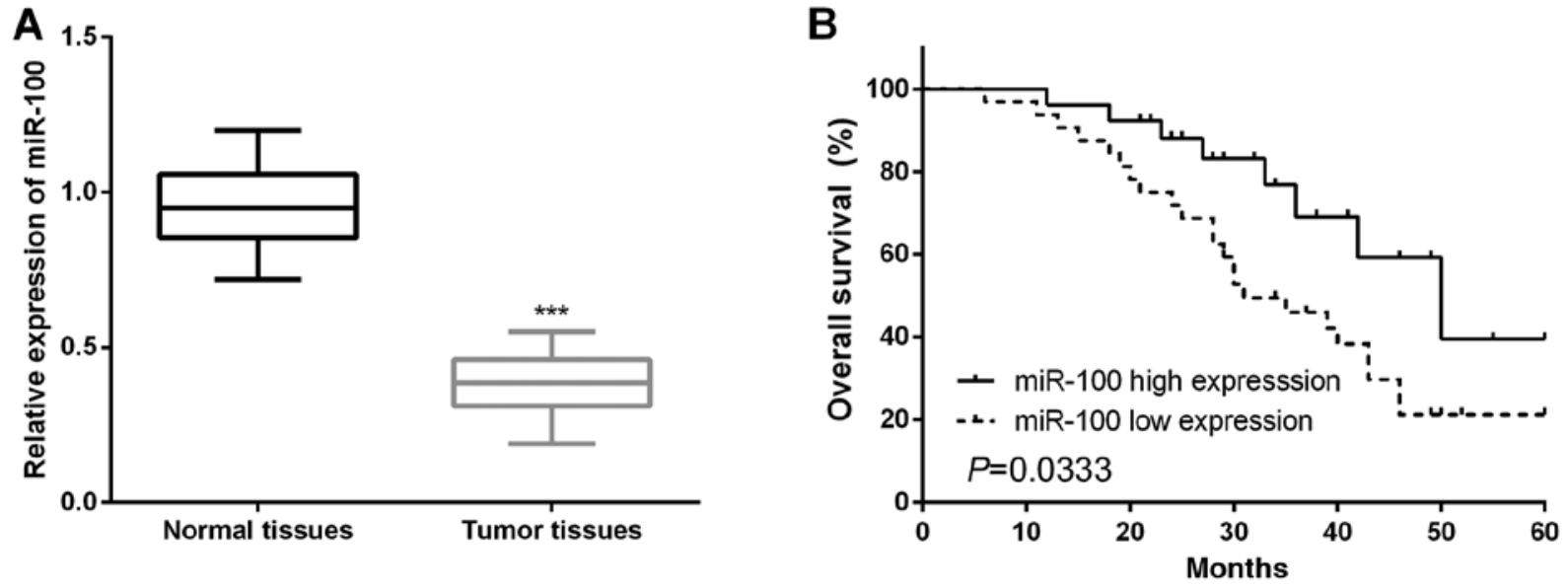

Figure 1. Decreased miR-100 expression was confirmed using qRT-PCR in CC tissues which were associated with poorer prognosis of CC patients. (A) qRT-PCR analysis was carried out to detect the miR-100 expression in CC tissue samples. (B) The Kaplan-Meier analysis demonstrated that patients with low miR-100 expression had poor OS. ${ }^{* * *} \mathrm{P}<0.001$. CC, cervical cancer.

plates for $8 \mathrm{~h}$ and treated with DHC for another $24 \mathrm{~h}$, and then incubated with $10 \mu \mathrm{g}$-ml BrdU (Sigma-Aldrich; Merck KGaA) for $0.5 \mathrm{~h}$. Each experiment was independently performed three times, and a two-tailed unpaired Student's t-test was performed to analyze the significance.

Transwell assays. Transwell chamber $(8.0 \mu \mathrm{m}$ pore size; Corning Co.) precoated with or without Matrigel (BD Biosciences) was utilized to perform invasion and migration assays. $\mathrm{CC}$ cells with different transfections in serum-free medium were seeded into the top chamber. Medium containing $10 \%$ FBS, as a chemoattractant, was added into the bottom chambers. Followed by incubation at $37^{\circ} \mathrm{C}$ with $5 \% \mathrm{CO}_{2}$ for $48 \mathrm{~h}$, cells remaining on the top surface were scraped away while cells attached to the bottom surface were fixed, stained and detected under an inverted microscope (Olympus Corporation) from five randomly selected visual fields.

Western blot analysis. Ice-cold Protein Lysis Buffer (Beyotime) supplemented with protease inhibitor cocktail (Sigma-Aldrich; Merck KGaA) was used to lyse the CC cells. Protein concentrations were determined using a BCA Protein assay kit (Beyotime) following the manufacturer's instructions. Proteins were separated with $10 \%$ sodium dodecyl sulfatepolyacrylamide gel electrophoresis (SDS-PAGE), followed by transferred onto PVDF membrane (Millipore) which was blocked in TBST with 5\% skim milk for $2 \mathrm{~h}$ at room temperature. Then, the membrane was incubated with the following primary antibodies overnight at $4^{\circ} \mathrm{C}$ : SATB1 $(1: 1,000$; ab109122), PI3K (1:1,000, ab191606) (both from Abcam), p-PI3K (1:1,000, BS4605; Xinle Biotechnology), Akt (1:1,000, sc-56878), p-Akt (1:1,000, sc-81433) (both from Santa Cruz Biotechnology, Inc.), mTOR (1:1000, ab32028), p-mTOR (1:1,000, ab109268), E-cadherin (1:1,000, ab133597), Vimentin (1:1,000, ab137321) and GAPDH (1:10,000, ab128915) (all from Abcam). The membranes were washed three times in TBST incubated with HRP-conjugated secondary antibody (1:2,000, ab205718; Abcam) at room temperature for $2 \mathrm{~h}$ and then washed with TBST. ECL reagents (Thermo Fisher Scientifc, Inc.) were utilized to visualize the proteins.
Dual-luciferase reporter assays. The wild-type (WT) and mutant (MUT) SATB1 3'-UTR were designed and prepared by GenePharma Co., Ltd., and were inserted into pGL3 plasmids (Promega). Subsequently, CC cells were cotransfected with either the SATB1-3'-UTR-MUT or SATB1-3'-UTR-WT reporter vector, along with miR-100 mimics by Lipofectamine 2000 (Invitrogen; Thermo Fisher Scientific, Inc.). After incubation for $48 \mathrm{~h}$, the dual-luciferase reporter assay system (Promega) was used to determine the luciferase activity in transfected cells according to the manufacturer's recommendations.

Xenograft tumor formation assay. This study was approved by the Ethics Committees on Animal Research of the Second Hospital of Shandong University. Female nude mice (4-6 week-old) were randomly divided into two groups. The HeLa cells infected with lentivirus containing lentiviral miR-100 (lenti-miR-100) or the negative control (lenti-control) were injected subcutaneously into right flank of mice. The size of tumors was measured every 3 days. The tumor volumes were calculated as follows: Volume $=\left(\right.$ length $\mathrm{x}$ width $\left.{ }^{2}\right)-2$. The mice were sacrificed at the end of the observation periods to excise the tumors.

Statistical analysis. All assays were repeated at least 3 times. All statistical analysis was carried out using SPSS software version 17.0 (SPSS Inc.). Student's t-test and one-way ANOVA followed by Tukey's post hoc test were utilized to analyze 2 or multiple groups. Kaplan-Meier curve and log-rank test were utilized to analyze the overall survival of $\mathrm{CC}$ patients. $\mathrm{P}<0.05$ indicates statistically significant difference.

\section{Results}

Low miR-100 expression level predicts poor prognosis of $C C$ patients. To elucidate the functions of miR-100 in CC progression, we detected the miR-100 expression in $\mathrm{CC}$ tissue specimens and matched normal tissue samples by performing qRT-PCR. It was clear that the expression of miR-100 in CC tissues was prominently decreased in comparison with that in paired healthy tissue samples (Fig. 1A). Moreover, the 
Table II. Correlation of miR-100 expression with the clinicopathological characteristics of the cervical cancer patients.

\begin{tabular}{|c|c|c|c|c|}
\hline \multirow{2}{*}{$\begin{array}{l}\text { Clinicopathological } \\
\text { features }\end{array}$} & \multirow[b]{2}{*}{ Cases $(\mathrm{n}=58)$} & \multicolumn{2}{|c|}{ miRNA-100 a expression } & \multirow[b]{2}{*}{ P-value } \\
\hline & & $\operatorname{High}(\mathrm{n}=21)$ & Low $(\mathrm{n}=37)$ & \\
\hline Age (years) & & & & 0.4128 \\
\hline$>60$ & 30 & 9 & 21 & \\
\hline$\leq 60$ & 28 & 12 & 16 & \\
\hline Family history of cancer & & & & 0.4651 \\
\hline Yes & 28 & 8 & 20 & \\
\hline No & 30 & 13 & 17 & \\
\hline Tumor size $(\mathrm{cm})$ & & & & 0.0921 \\
\hline$\geq 5.0$ & 26 & 7 & 19 & \\
\hline$<5.0$ & 32 & 14 & 18 & \\
\hline TNM stage & & & & $0.0018^{\mathrm{b}}$ \\
\hline I-II & 27 & 17 & 10 & \\
\hline III & 31 & 4 & 27 & \\
\hline Lymph node metastasis & & & & $0.0025^{\mathrm{b}}$ \\
\hline Yes & 16 & 5 & 11 & \\
\hline No & 42 & 16 & 26 & \\
\hline Menopause & & & & 0.2468 \\
\hline Yes & 27 & 10 & 17 & \\
\hline No & 32 & 11 & 20 & \\
\hline FIGO stage & & & & $0.0031^{\mathrm{b}}$ \\
\hline I-II & 26 & 17 & 9 & \\
\hline III-IV & 32 & 4 & 28 & \\
\hline Distant metastasis & & & & 0.4396 \\
\hline Yes & 29 & 7 & 22 & \\
\hline No & 29 & 14 & 15 & \\
\hline HPV infection & & & & 0.4952 \\
\hline Negative & 30 & 11 & 20 & \\
\hline Positive & 28 & 10 & 17 & \\
\hline
\end{tabular}

${ }^{a}$ The mean expression level of miR-100 was used as the cutoff; ${ }^{b}$ statistically significant. TNM, tumor-node-metastasis; FIGO, International Federation of Gynecology and Obstetrics.

association between miR-100 expression and the clinicopathological features of CC patients was explored by classifying the $\mathrm{CC}$ patients into two groups on the basis of mean expression of miR-100. As presented in Table II, lower miR-100 expression was notably associated with poorer clinicopathologic features of CC patients. The association between miR-100 and the OS of CC patients was determined via Kaplan-Meier analysis and the log-rank test. Results showed that CC patients who had lower miR-100 expression presented poorer OS compared with patients with higher miR-100 expression (Fig. 1B).

Restoration of miR-100 significantly repressed CC cell proliferation. For a better understanding of the functions of miR-100 in CC development, the effects of miR-100 on CC cell proliferation was investigated. qRT-PCR was carried out to detect the miR-100 expression in CC cells and normal cervical epithelial cells. The results demonstrated significant decrease of miR-100 expression in CC cells compared with the Ect1-E6E7 cells (Fig. 2A). Subsequently, due to the relatively low or high endogenous miR-100 expression, HeLa and
Ca-Ski cells were selected to study the potential functions and mechanisms of miR-100. The miR-100 expression was altered by transfecting miR-100 mimics or inhibitor into HeLa and Ca-Ski cells and qRT-PCR was carried out to examine the efficiency. miR-100 was dramatically overexpressed by miR-100 mimics in HeLa cells, whereas, obviously downregulated by miR-100 inhibitor in Ca-Ski cells (Fig. 2B and C). Then, MTT assay was conducted to investigate the influence of miR-100 on CC cell proliferation ability. Data indicated that miR-100 overexpression prominently inhibited CC cell proliferation while miR-100 inhibition markedly enhanced the proliferation capacity (Fig. 2D and E). To further explore the potential function of miR-100 in cervical cancer cells, BrdU assay was performed to assess the effects of miR-100 on HeLa and Ca-Ski cells. Compared to controls, miR-100 mimics resulted in a decrease in BrdU positive cells, whereas, miR-100 inhibitor increased BrdU positive cells (Fig. 2F and G). This observation indicates that miR-100 has a stimulating effect on the proliferation of HeLa and Ca-Ski cells. 
A

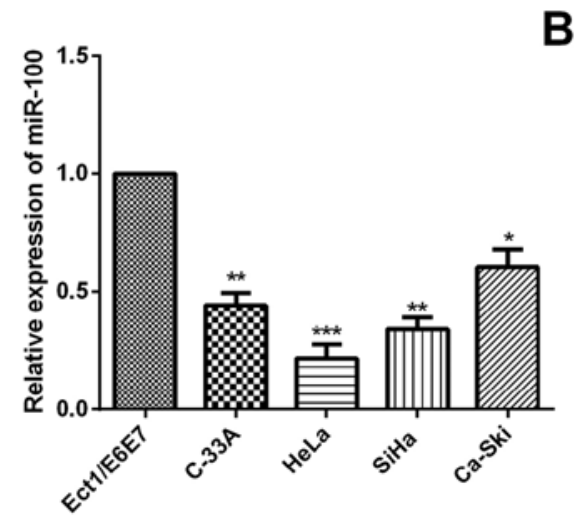

B

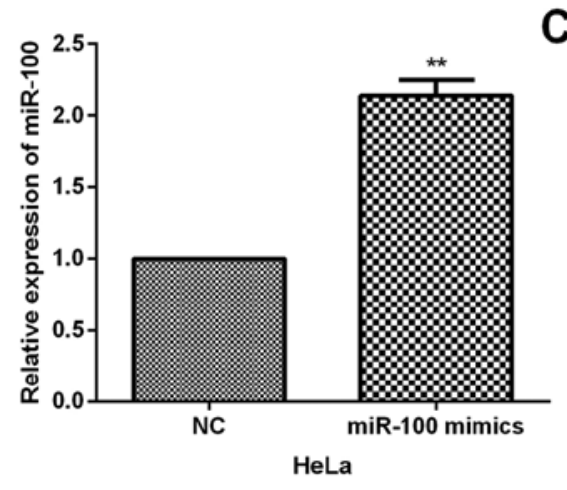

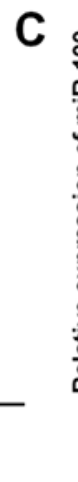

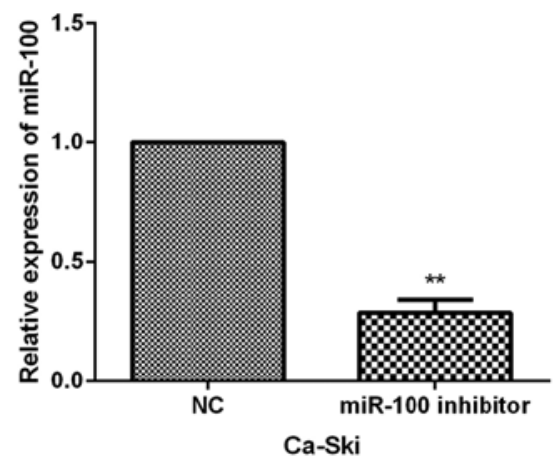

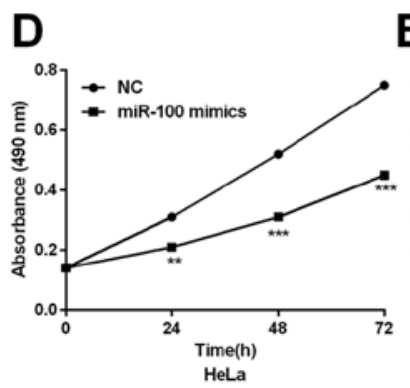
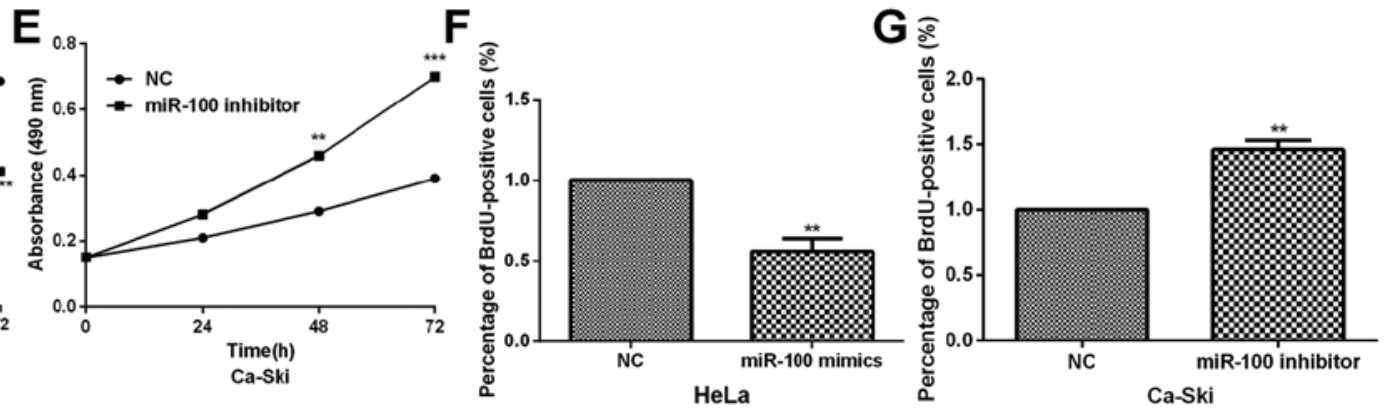

Figure 2. miR-100 overexpression inhibits CC cell proliferation. (A) miR-100 expressions in CC cells was determined by qRT-PCR. (B) miR-100 expression in miR-100-overexpressed HeLa cells. (C) miR-100 expression in miR-100-suppressed Ca-Ski cells. (D and E) The proliferation abilities of HeLa or Ca-Ski cells treated with miR-100 mimics or inhibitor were determined by MTT assays. (F and G) BrdU assay was used to evaluate cell proliferation in HeLa or Ca-Ski cells. ${ }^{*} \mathrm{P}<0.05,{ }^{* *} \mathrm{P}<0.01$ and ${ }^{* * *} \mathrm{P}<0.001$. CC, cervical cancer; BrdU, 5-bromo-2'-deoxyuridine.

miR-100 overexpression notably suppresses CC cell invasion and migration. To further confirm whether miR-100 could regulate the $\mathrm{CC}$ progression and metastasis, Transwell assays were performed and results indicated that miR-100 overexpression dramatically repressed HeLa cell invasion and migration abilities (Fig. 3A and B). In contrast, the inhibition of miR-100 in Ca-Ski cells prominently promoted the invasion and migration capacities (Fig. 3C and D). These results revealed that miR-100 played inhibitory roles in CC progression.

SATB1 is a functional target of miR-100 in CC cells. To explore the underlying mechanism of the functional roles of miR-100 in CC, the putative targets of miR-100 were analyzed and target scan analysis suggested that SATB1 was a potential target for miR-100 (Fig. 4A). Luciferase reporter assay was carried out to determine the interaction between miR-100 and SATB1. Results demonstrated that miR-100 overexpression prominently declined the luciferase activity of SATB1-3'-UTR-WT; on the other hand, miR-100 mimics did not change the luciferase activity of CC cells with SATB1-3'-UTR-MUT (Fig. 4B). In addition, to investigate whether miR-100 had effects on SATB1 expression, qRT-PCR analysis was performed to measure the SATB1 expression in CC cells with transfections of miR-100 mimics or inhibitor. Data indicated that resumption of miR-100 evidently suppressed the SATB1 expression in HeLa cells whereas miR-100 inhibition obviously enhanced the SATB1 expression in Ca-Ski cells (Fig. 4C and D).

miR-100 regulates AKT/mTOR signaling pathway and $E M T$ in CC cells. As we verified that SATB1 was a target for miR-100, the expression of SATB1 in CC tissues was measured by IHC assays. As shown in Fig. 5A and B, the findings revealed that SATB1 mainly localized at the nucleus and was dramatically upregulated in CC tissue samples. In addition, Kaplan-Meier analysis further indicated that CC patients with relatively higher SATB1 level had significantly decreased OS (Fig. 5C). Moreover, to clarify the specific molecular mechanisms under miR-100 suppressing CC cell progression, our studies were extended on AKT/mTOR signaling pathway and EMT. Western blot analysis demonstrated that expression of p-AKT and p-mTOR was obviously inhibited by miR-100 overexpression in HeLa cells while there was no prominent variation in AKT and mTOR expression; whereas, miR-100 inhibition notably increased the p-AKT and p-mTOR expression in Ca-Ski cells (Fig. 5D). As EMT is a pivotal progress in tumor cell metastasis and invasion, the functions of miR-100 in CC cell EMT was analyzed by detecting the protein levels of EMT markers. Results demonstrated that the E-cadherin expression was obviously increased while the $\mathrm{N}$-cadherin and vimentin expression was significantly decreased in miR-100 overexpressed HeLa cells. Whereas, suppression of miR-100 reversed these results (Fig. 5D). Overall, these results implied that miR-100 inhibited CC progression by regulation of AKT/mTOR signaling pathway and EMT.

miR-100 inhibits CC cell tumor growth in vivo. In the present study, we further investigated the effects of miR-100 on CC tumor growth in vivo. HeLa cells were stably transfected with lenti-miR-100 or the negative control. Tumor volume analysis indicated that the volume of mice in lenti-miR-23b 

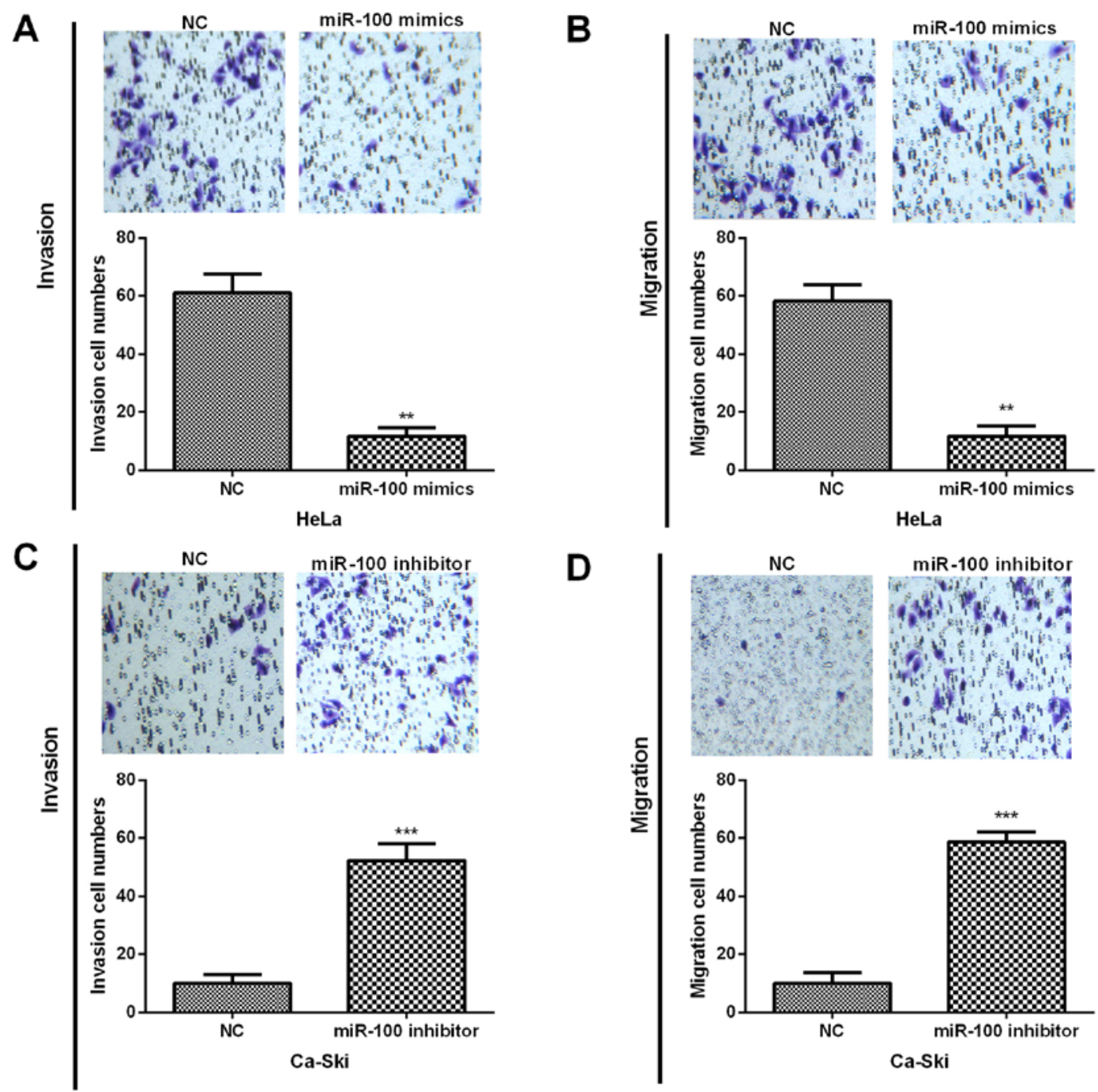

Figure 3. miR-100 restoration significantly represses CC cell invasion and migration. (A and B) The invasion and migration abilities of HeLa cells treated with miR-100 mimics were assessed by Transwell assays. (C and D) Transwell assay was performed to determine the invasion and migration capacities of Ca-Ski cells treated with miR-100 inhibitor. ${ }^{* *} \mathrm{P}<0.01$ and ${ }^{* * *} \mathrm{P}<0.001$. CC, cervical cancer.

\section{A}

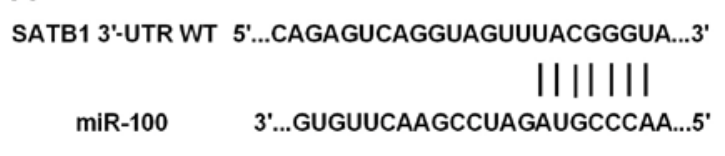

SATB1 3'-UTRMUT 5'...CAgAgucAgguaguUCGAUUUCA...3'

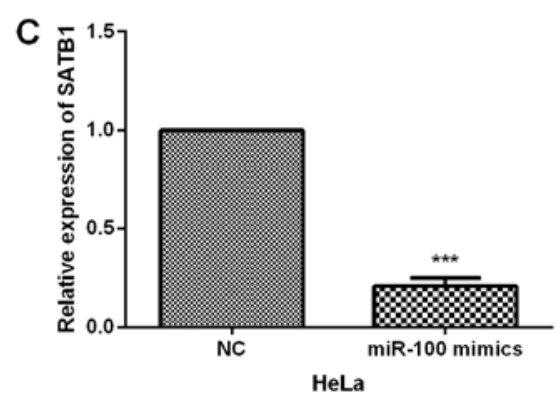

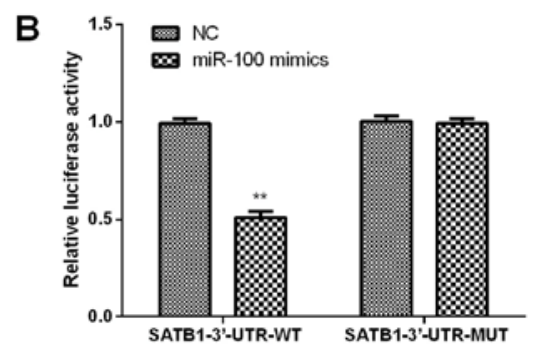

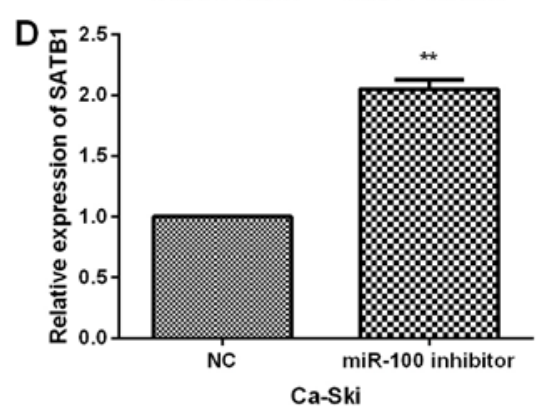

Figure 4. SATB1 is a target for miR-100 in CC cells. (A) Predicted binding sequences of miR-100 in SATB1 3'-UTR. (B) Luciferase reporter assays were conducted to detect the luciferase activities of CC cells cotransfected with SATB1 3'-UTR-WT or -MUT and miR-100 mimics. (C and D) SATB1 expression in HeLa or Ca-Ski cells treated with miR-100 mimics or inhibitor. ${ }^{* *} \mathrm{P}<0.01$ and ${ }^{* * *} \mathrm{P}<0.001$. CC, cervical cancer. SATB1, special AT-rich sequence-binding protein 1; 3'-UTR, 3'-untranslated region; WT, wild-type, MUT, mutant. 


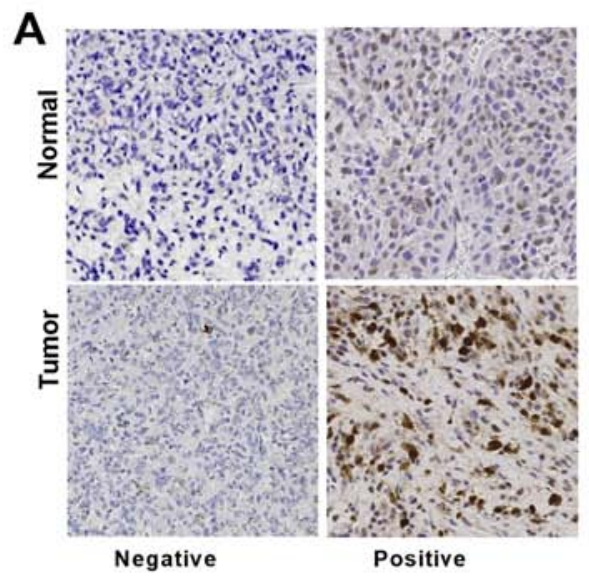

B

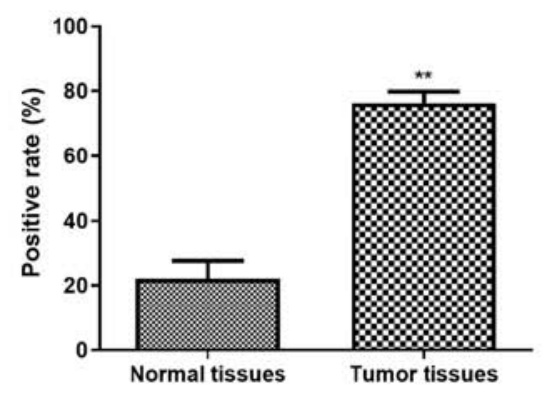

C

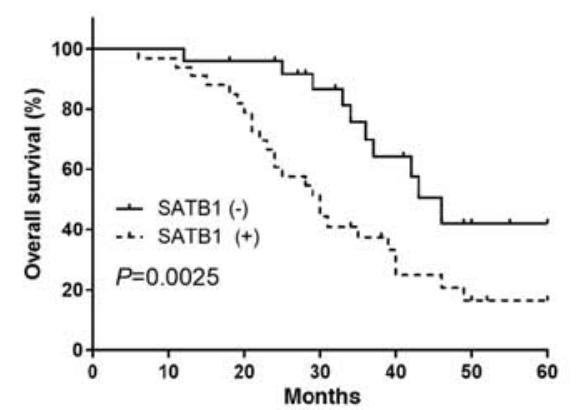

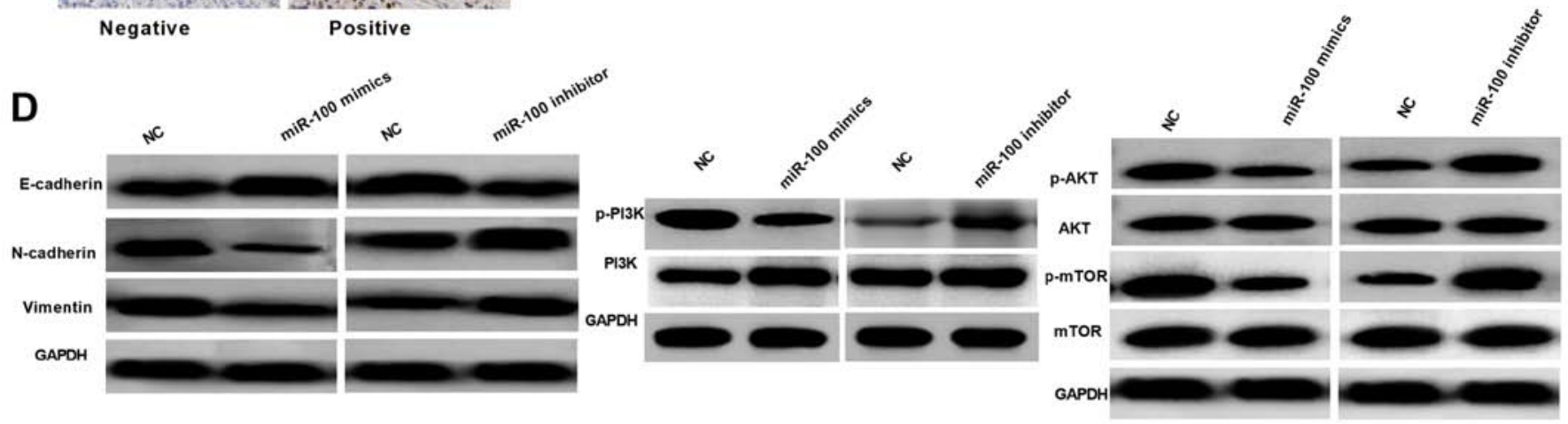

Figure 5. miR-100 modulates the EMT progress and AKT/mTOR signaling pathway in CC cells. (A and B) SATB1 expression in CC tissues was measures by performing IHC assays. (C) Kaplan-Meier analysis revealed that CC patients with high SATB1 expression had poor OS. (D) miR-100 regulated AKT/mTOR signaling pathway and EMT in CC cells. ${ }^{* *} \mathrm{P}<0.01$. CC, cervical cancer; EMT, epithelial-to-mesenchymal transition; SATB1, special AT-rich sequence-binding protein 1.

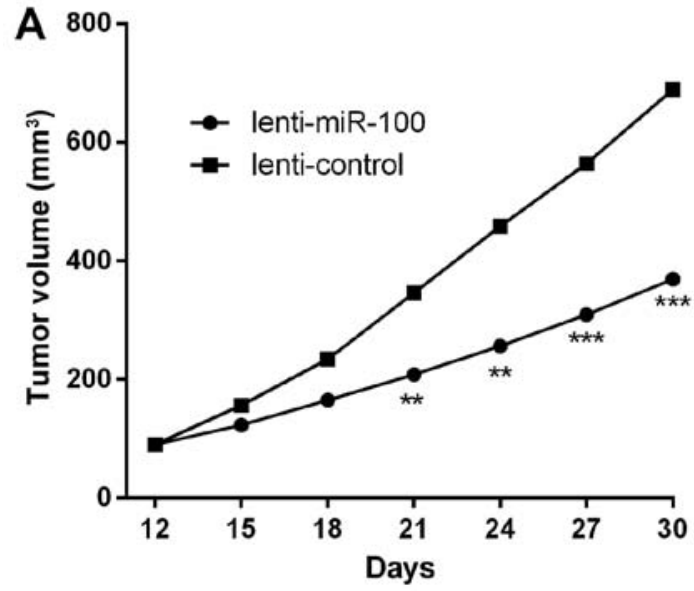

B

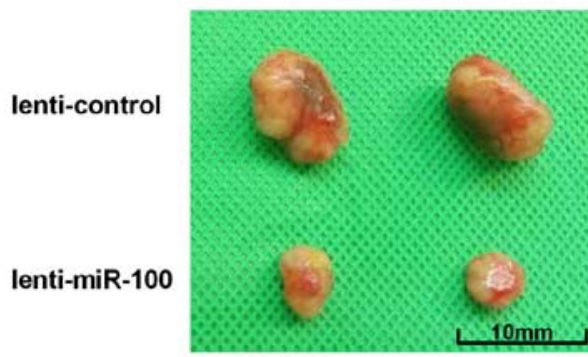

Figure 6. miR-100 restoration notably suppressed CC tumorigenesis in vivo. (A) Representative images of CC xenograft tissues in different groups. (B) The tumor volumes were measured every 3 days from day 12 to 30 . The two xenografts were replicates of an experiment, not two experiments. ${ }^{* *} \mathrm{P}<0.01$ and ${ }^{* * * *} \mathrm{P}<0.001$. CC, cervical cancer.

group were obviously smaller than the controls. In addition, the growth rate was significantly slower than the control groups (Fig. 6A and B).

\section{Discussion}

$\mathrm{CC}$ is currently the leading factor for deaths in women of childbearing ages, and existing conventional therapeutic screening approaches remain ineffective for advanced stage CC (21). Therefore, elucidating the mechanisms underlying CC progression and identifying biomarkers for prevention, early diagnosis and treatment of CC patients have become the hot spot of CC research (22). Emerging evidence has indicated that certain aberrantly expressed miRNAs are involved in tumorigenesis through regulating the expression of their targets and serve as novel biomarkers for the diagnosis and prognosis of different human malignancies, including CC (23). For example, Chen and Liu (24) reported that miR-744 inhibited CC growth and progression through inducing apoptosis via regulation of Bcl-2. Chen et al (25) confirmed that miR-206 and miR-34a functioned as novel prognostic and therapeutic biomarkers in CC. Hence, identification of miRNAs and their targets 
implicated in tumorigenesis may provide key clues to develop novel diagnostic methods and therapies for CC patients.

Previous studies demonstrated that miR-100 exerted tumor suppressive functions in numerous cancers by modulating different targets. For instance, Liu et al (26) proposed that miR-100 repressed cell proliferation, invasion and migration and promoted chemosensitivity in osteosarcoma via regulating IGFIR; Luan et al (27) proposed that miR-100 upregulation suppressed glioblastoma cell chemosensitivity, proliferation and migration through FGFR3; studies by Qureshi et al (28) demonstrated that miR-100 was a novel non-invasive biomarker for earlier diagnosis of bladder cancer. Thus, we assumed that miR-100 might serve as a cancer repressor in CC. To test the hypothesis, series of experiments was performed. Data revealed that miR-100 expression was obviously decreased in CC and the low miR-100 expression was related to its malignant progression and poor prognosis. In addition, functional experiments indicated that miR-100 restoration repressed the proliferation, invasion and migration capacities of CC cells via regulation of AKT/mTOR signaling pathway and EMT progress. Whereas, miR-100 overexpression was also able to inhibit CC tumorigenesis in vivo. These results demonstrated the potential tumor inhibitory roles of miR-100 in CC progression.

SATB1 has been confirmed to be implicated in various cellular processes, including invasion, migration, apoptosis and proliferation $(29,30)$. In the present study, the functional effects of SATB1 wewe further investigated on CC progression. Results in the present study successfully revealed that SATB1 was upregulated in CC, which demonstrated poor OS of CC patients. Moreover, SATB1 was confirmed to be a target for miR-100 in CC cells. Our findings illustrated that functions of miR-100 were regulated by SATB1. Thus, our results provided new insight into the miR-100 biological functions in $\mathrm{CC}$ cells.

Collectively, evidence was provided that miR-100 exerted an inhibitory role in $\mathrm{CC}$ progression both in vitro and in vivo. It was confirmed that miR-23b expression was notably declined in $\mathrm{CC}$ tissues and cell lines, whereas, the low miR-23b expression was related to poor OS and worse prognosis. The functional assays indicated that miR-23b overexpression prominently decreased the proliferation, invasion and migration abilities of CC cells through regulation of the AKT/mTOR signaling pathway and EMT. Moreover, SATB1 was identified as one functional target for miR-23b in CC cells. Hence, the findings of the present study provide a new clue to the functions of miR-23b/SATB1 in CC progression as novel diagnostic and prognosis markers.

\section{Acknowledgements}

Not applicable.

\section{Funding}

No funding was received.

\section{Availability of data and materials}

The datasets used and/or analyzed during the current study are available from the corresponding author on reasonable request.

\section{Authors' contributions}

$\mathrm{CH}, \mathrm{XQ}$ and NZ participated in the conception and design of the study, and performed the experiments. $\mathrm{HJ}$ performed the analysis and interpretation of the data. SZ wrote the manuscript, assisted with the statistical analysis of the data and contributed with constructive discussions. HY was involved in the conception of the study and provided the patients' clinical data as well as crucial experimental materials. All authors read and approved the final version of the manuscript.

\section{Ethics approval and consent to participate}

The present study was approved by the Ethics Committee of the Second Hospital of Shandong University (Jinan, China), and the Ethics Committee on Animal Research of Shandong University. All patients involved in the present study provided written informed consent.

\section{Patient consent for publication}

Not applicable.

\section{Competing interests}

The authors declare that they have no competing interests.

\section{References}

1. Siegel RL, Miller KD and Jemal A: Cancer statistics, 2016. CA Cancer J Clin 66: 7-30, 2016.

2. Carr KR, Ioffe YJ, Filippova M, Duerksen-Hughes P and Chan PJ: Combined ultrasound-curcumin treatment of human cervical cancer cells. Eur J Obstet Gynecol Reprod Biol 193: 96-101, 2015

3. Yu Q, Yang X, Duan W, Li C, Luo Y and Lu S: miRNA-346 promotes proliferation, migration and invasion in liver cancer. Oncol Lett 14: 3255-3260, 2017.

4. Puik JR, Meijer LL, Le Large TY, Prado MM, Frampton AE, Kazemier $\mathrm{G}$ and Giovannetti E: miRNA profiling for diagnosis, prognosis and stratification of cancer treatment in cholangiocarcinoma. Pharmacogenomics 18: 1343-1358, 2017.

5. Fang H, Xie J, Zhang M, Zhao Z, Wan Y and Yao Y: miRNA-21 promotes proliferation and invasion of triple-negative breast cancer cells through targeting PTEN. Am J Transl Res 9: 953-961, 2017.

6. Xiao R, Li C and Chai B: miRNA-144 suppresses proliferation and migration of colorectal cancer cells through GSPT1. Biomed Pharmacother 74: 138-144, 2015.

7. Yang L, Liang H, Wang Y, Gao S, Yin K, Liu Z, Zheng X, Lv Y, Wang L, Zhang CY, et al: MiRNA-203 suppresses tumor cell proliferation, migration and invasion by targeting Slug in gastric cancer. Protein Cell 7: 383-387, 2016.

8. Deng B, Zhang S, Zhang Y, Miao Y, Meng X and Guo K: Knockdown of Tripartite Motif Containing 28 suppresses the migration, invasion and epithelial-mesenchymal transition in ovarian carcinoma cells through downregulation of Wnt/ $\beta$-catenin signaling pathway. Neoplasma 64: 893-900, 2017.

9. Wu HY and Cai XP: miR-338-3p suppresses epithelial-mesenchymal transition and metastasis in human nonsmall cell lung cancer. Indian J Cancer 52 (Suppl 3): E168-E171, 2015.

10. Chen $\mathrm{H}$, Jia W and Li J: ECM1 promotes migration and invasion of hepatocellular carcinoma by inducing epithelial-mesenchymal transition. World J Surg Oncol 14: 195, 2016.

11. Colditz J, Rupf B, Maiwald C and Baniahmad A: Androgens induce a distinct response of epithelial-mesenchymal transition factors in human prostate cancer cells. Mol Cell Biochem 421: 139-147, 2016.

12. He H, Dai J, Xu Z, He W, Wang X, Zhu Y and Wang H: Fbxw7 regulates renal cell carcinoma migration and invasion via suppression of the epithelial-mesenchymal transition. Oncol Lett 15: 3694-3702, 2018. 
13. Chen X, Liao Y, Yu Y, Zhu P, Li J, Qin L, Liao W and Huang Z: Elevation of MAP17 enhances the malignant behavior of cells via the Akt/mTOR pathway in hepatocellular carcinoma. Oncotarget 8: 92589-92603, 2017.

14. Xue L, Wang Y, Yue S and Zhang J: Low MiR-149 expression is associated with unfavorable prognosis and enhanced Akt/mTOR signaling in glioma. Int J Clin Exp Pathol 8: 11178-11184, 2015.

15. Cai S, Han HJ and Kohwi-Shigematsu T: Tissue-specific nuclear architecture and gene expression regulated by SATB1. Nat Genet 34: 42-51, 2003.

16. Qi H, Fu X, Li Y, Pang X, Chen S, Zhu X, Li F and Tan W: SATB promotes epithelial-mesenchymal transition and metastasis in prostate cancer. Oncol Lett 13: 2577-2582, 2017.

17. Li YC, Bu LL, Mao L, Ma SR, Liu JF, Yu GT, Deng WW, Zhang WF and Sun ZJ: SATB1 promotes tumor metastasis and invasiveness in oral squamous cell carcinoma. Oral Dis 23 : 247-254, 2017.

18. Pan Z, Jing W, He K, Zhang L and Long X: SATB1 is correlated with progression and metastasis of breast cancers: A meta-analysis. Cell Physiol Biochem 38: 1975-1983, 2016.

19. Hu XT, Chen W, Zhang FB, Shi QL, Hu JB, Geng SM and He C: Depletion of the proteasome subunit PSMA7 inhibits colorectal cancer cell tumorigenicity and migration. Oncol Rep 22: 1247-1252, 2009.

20. Hu XT, Chen W, Wang D, Shi QL, Zhang FB, Liao YQ, Jin M and $\mathrm{He} \mathrm{C}$ : The proteasome subunit PSMA7 located on the 20q13 amplicon is overexpressed and associated with liver metastasis in colorectal cancer. Oncol Rep 19: 441-446, 2008

21. Kessler TA: Cervical cancer: Prevention and early detection. Semin Oncol Nurs 33: 172-183, 2017.

22. Cordeiro MN, De Lima RC, Paolini F, Melo AR, Campos AP, Venuti A and De Freitas AC: Current research into novel therapeutic vaccines against cervical cancer. Expert Rev Anticancer Ther 18: 365-376, 2018.
23. Zhang Y, Zhang D, Wang F, Xu D, Guo Y and Cui W: Serum miRNAs panel (miR-16-2*, miR-195, miR-2861, miR-497) as novel non-invasive biomarkers for detection of cervical cancer. Sci Rep 5: 17942, 2015.

24. Chen XF and Liu Y: MicroRNA-744 inhibited cervical cancer growth and progression through apoptosis induction by regulating Bcl-2. Biomed Pharmacother 81: 379-387, 2016.

25. Chen AH, Qin YE, Tang WF, Tao J, Song HM and Zuo M: MiR-34a and miR-206 act as novel prognostic and therapy biomarkers in cervical cancer. Cancer Cell Int 17: 63, 2017.

26. Liu Y, Zhu ST, Wang X, Deng J, Li WH, Zhang P and Liu BS: MiR-100 inhibits osteosarcoma cell proliferation, migration, and invasion and enhances chemosensitivity by targeting IGFIR. Technol Cancer Res Treat 15: NP40-NP48, 2016.

27. Luan Y, Zhang S, Zuo L and Zhou L: Overexpression of miR-100 inhibits cell proliferation, migration, and chemosensitivity in human glioblastoma through FGFR3. Onco Targets Ther 8: 3391-3400, 2015

28. Qureshi A, Fahim A, Kazi N, Farsi Kazi SA and Nadeem F: Expression of miR-100 as a novel ancillary non-invasive biomarker for early detection of bladder carcinoma. J Pak Med Assoc 68: 759-763, 2018.

29. Xiao T, Fu L and Jie Z: SATB1 overexpression correlates with gastrointestinal neoplasms invasion and metastasis: A meta-analysis for Chinese population. Oncotarget 8: 48282-48290, 2017.

30. Huang B, Xiong F, Wang S, Lang X, Wang X and Zhou H: Effect of SATB1 silencing on the proliferation, invasion and apoptosis of TE-1 esophageal cancer cells. Oncol Lett 13: 2915-2920, 2017.

This work is licensed under a Creative Commons

Attribution-NonCommercial-NoDerivatives 4.0 International (CC BY-NC-ND 4.0) License. 